\title{
Ageing disrupts reinforcement learning whilst learning to help others is preserved
}

Jo Cutlerª,b,c, Marco Wittmanna,b, Ayat Abdurahmanª,b,d, Luca Hargitaia, Daniel Drew $^{a, b}$, Masud Husaina,b, Patricia Lockwood*a,b,c

aDepartment of Experimental Psychology, University of Oxford, United Kingdom bWellcome Centre for Integrative Neuroimaging, Department of Experimental Psychology, University of Oxford, United Kingdom

'Centre for Human Brain Health, School of Psychology University of Birmingham, United Kingdom

dDepartment of Psychology, University of Cambridge, United Kingdom

*Correspondence should be addressed to:

Jo Cutler: J.L.Cutler@bham.ac.uk

Centre for Human Brain Health, Birmingham University, Edgbaston Park Road, Birmingham, B15 2TT, United Kingdom.

Patricia Lockwood: patricia.lockwood@psy.ox.ac.uk

Department of Experimental Psychology, University of Oxford, Tinsley Building, Mansfield Road, Oxford, United Kingdom OX1 3SR. 


\section{Abstract}

2

3 Reinforcement learning is a fundamental mechanism displayed by many species.

4 However, adaptive behaviour depends not only on learning about actions and

5 outcomes that affect ourselves, but also those that affect others. Here, using

6 computational reinforcement learning models, we tested whether young (age 18-36)

7 and older (age 60-80, total $n=152$ ) adults can learn to gain rewards for themselves,

8 another person (prosocial), or neither individual (control). Detailed model comparison

9 showed that a model with separate learning rates for each recipient best explained

10 behaviour. Young adults were faster to learn when their actions benefitted themselves,

11 compared to helping others. Strikingly, compared to younger adults, older adults

12 showed preserved prosocial learning rates but reduced self-relevant learning rates.

13 Moreover, psychopathic traits were lower in older adults and negatively correlated with

14 prosocial learning. These findings suggest learning how to benefit others is preserved

15 across the lifespan with implications for reinforcement learning and theories of healthy 16 ageing.

17

18 Keywords: Prosocial behaviour, ageing, reinforcement learning, computational 19 modelling 
Learning associations between actions and their outcomes is fundamental for adaptive behaviour. To date, the majority of studies examining reinforcement learning have tested how we learn associations between actions and outcomes that affect ourselves, and largely focused on these processes in young age, both in humans and other species $^{1-5}$. However, such self-relevant learning may be computationally separable from learning about actions that help other people. Studies suggest slower learning of associations between actions and outcomes when they are about ${ }^{6}$ or affect others ${ }^{7}$, henceforth referred to as 'prosocial learning'.

Senescence is associated with a multitude of changes including declines in cognitive functioning and perception, but perhaps preservation of affective processing and social cognitive abilities ${ }^{8-10}$. However, less is known about how ageing affects social behaviour, despite the critical importance of this question. Social isolation has been found to be as damaging to physical health as smoking or excessive drinking ${ }^{11}$. Social behaviours that benefit others - prosocial behaviours - are vital for maintaining social bonds and relationships ${ }^{12}$ across the lifespan. In addition to the benefits for others, prosociality has been linked to improved life satisfaction ${ }^{13}$, mental wellbeing ${ }^{14}$, and physical health ${ }^{15}$ for the person being prosocial, all of which could contribute to healthy ageing. A key aspect of prosocial behaviour is the ability to learn associations between our own actions and outcomes for other people?. Here, we use computational models of reinforcement learning in young and older participants to examine the mechanisms that underpin self-relevant and prosocial learning and associations with healthy individual differences in socio-cognitive ability.

Reinforcement Learning Theory $(R L T)$ provides a powerful framework for understanding and precisely modelling learning ${ }^{16}$. In $R L T$, prediction errors signal the unexpectedness of outcomes and affect the choices we make in the future. The influence that prediction errors have on choices can be modelled individually through the learning rate, which quantifies the effect of past outcomes on subsequent behaviour. The plausibility of reinforcement learning as a core biological mechanism for action-outcome associations is bolstered by our understanding of neurobiology, with prediction errors encoded by single neurons in the ventral tegmental area ${ }^{17}$. 
52 Although essential for successful adaptive behaviour, several studies suggest that our propensity for reinforcement learning declines in later life ${ }^{10}$. Compared to younger adults, older adults show learning impairments particularly when action-outcome associations are probabilistic ${ }^{18}$ or reverse ${ }^{19}$. Age-related declines in learning ability have been linked to functional and structural changes in frontostriatal circuits ${ }^{20,21}$ and dopamine transmission ${ }^{18,22}$, which shows a significant age-related decrease ${ }^{23-25}$ and has a key role in coding prediction errors $2,26,27$. Indeed, one study showed that administering L-DOPA, a dopamine precursor, to older adults increased their learning rate $^{28}$. Therefore, if reinforcement learning in general declines in older age, we would hypothesise lower learning rates for both self-relevant and prosocial learning in older, compared to younger, adults.

Alternatively, prosocial learning may depend not only on our learning ability, but also our motivation to help others. Results from experiments using economic games to measure prosociality have found that older adults tend to be more generous ${ }^{29,30}$. There is also evidence of an age-related increase in charitable donations to individuals in need $^{31}$. At work, older adults engage in more prosocial behaviours than younger adults, according to both self-report data and colleagues' ratings ${ }^{32}$. Finally, selfreported altruism and decisions to donate to others have been shown to increase with age $^{33}$. However, one limitation of these studies is that the paradigms often place self and other reward preferences in conflict. Money for the other person depends on less money for oneself. Moreover, older adults generally have higher accumulated wealth, which would be an important confound in studies of monetary exchange ${ }^{34}$. Prosocial learning avoids this confound by separating outcomes for oneself from outcomes for others. If older adults do indeed value outcomes for others more than young adults, we might expect that whilst self-relevant learning declines with ageing, prosocial learning could be preserved. Comparing young and older adults on self-relevant and prosocial learning provides an opportunity to dissociate how possible age-related changes in cognitive ability and social behaviour impact on learning.

While studies point to potential group differences between young and older adults, 83 there is also substantial variability in self and other reward sensitivity. For example, 
psychopathy is a key trait associated with decreased prosocial behaviour and altered self and other reward processing ${ }^{35,36}$. Psychopathy has dysfunctional affectiveinterpersonal features at its core $\mathrm{e}^{37,38}$ but is also characterised by lifestyle and antisocial traits ${ }^{39}$. At the extreme, psychopathy is a severe personality condition linked to poor life outcomes, violence, and criminality ${ }^{40-42}$. However, several studies suggest similar behavioural and neural profiles between community samples with high levels of psychopathic traits ${ }^{43}$ and those with clinical diagnoses of psychopathy, consistent with the Research Domains of Criteria (RDoC) conceptualisation of a dimensional approach to psychiatry ${ }^{44}$. This $\mathrm{RDoC}$ approach suggests that psychiatric disorders can be thought of as dimensional, rather than categorical, constructs. In a similar vein, psychopathic traits can be captured on a continuum spanning clinical samples and the general population, with a range of scores on that continuum for healthy people. Selfreport measures of psychopathic traits that mirror the latent structure of clinical psychopathy measures, comprising antisocial and interpersonal dimensions, are available to use in samples from the general population ${ }^{39}$.

Intriguingly, preliminary evidence suggests that ageing may also be associated with changes in psychopathic traits, which could have important implications for our understanding of an ageing population. Epidemiological studies show that criminal activity increases during adolescence then declines in older adulthood ${ }^{45}$. Antisocial and aggressive behaviours also significantly decrease in older age, with young adults (age 16-24 years) having the highest rates of homicide ${ }^{46}$. Even within violent male offenders, psychopathic traits linked to an antisocial lifestyle are negatively correlated with age ${ }^{47}$. In community samples, ageing is associated with a decrease in both the antisocial-lifestyle (antisocial and impulsive behaviours) and affective-interpersonal (lack of empathy and guilt) elements of psychopathic traits ${ }^{48}$. These studies highlight the importance of assessing how differences in psychopathic traits could map on to differences in prosocial behaviours. However, no existing work has examined this question.

114 Taken together, previous research supports opposing hypotheses for how ageing is associated with self-relevant and prosocial reinforcement learning. On the one hand, 
116

evidence suggests that older adults should be impaired at learning, regardless of the recipient, consistent with ageing-related declines in learning ability and dopamine transmission. On the other hand, potential increases in valuing outcomes for others in older, compared to younger, adults would predict preserved prosocial learning ability but reduced self-relevant learning ability. Finally, we expected variation in psychopathic traits to be associated with learning for others but not self in both age groups.

To distinguish between these competing hypotheses, we tested 75 young (aged 1836, mean=23.07, 44 females) and 77 older (aged 60-80, mean=69.84, 40 females) adults carefully matched on gender, years of education, and IQ. Participants completed a probabilistic reinforcement-learning task (Figure 1) designed to separate self-relevant (rewards for self) from prosocial learning (rewards for another person), as well as controlling for the general valence of receiving positive outcomes (rewards for neither self or other).

Detailed model comparison showed that a computational model with separate learning rates best explained how people learn associations for different recipients (Figure 2). Young adults were faster to learn when their actions benefitted themselves, compared to when they helped others. Strikingly however, older adults showed a reduced selfbias, with a relative increase in the rate at which they learnt about actions that helped others, compared to themselves (Figure $3 a \& b$ ). Older adults had significantly reduced levels of psychopathic traits compared to younger adults and in older adults, lower psychopathic trait scores correlated with prosocial learning rates (Figure $4 a$ \& b). These effects were not explained by individual differences in IQ, memory or attention abilities. Overall, we show that older adults are less self-biased in reinforcement learning than young adults, and ageing is associated with a decline in psychopathic traits. These findings suggest learning how our actions help others is preserved across the lifespan. 
a

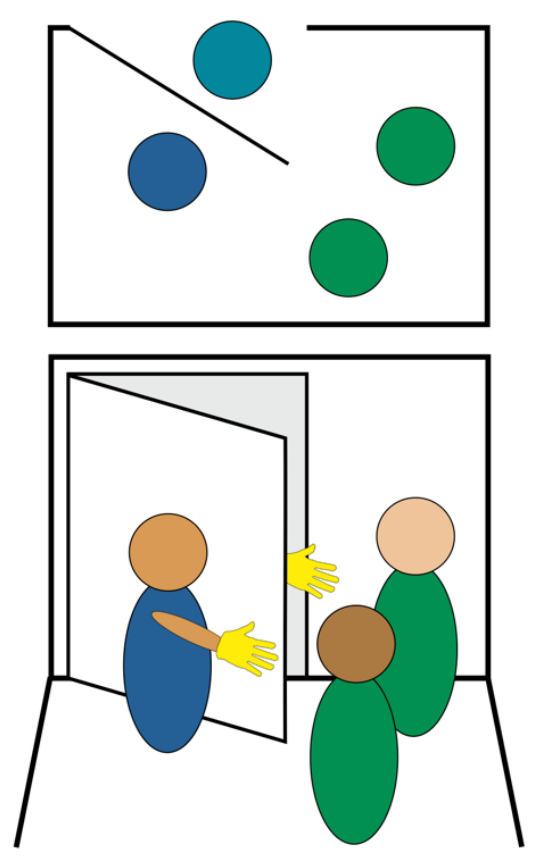

b

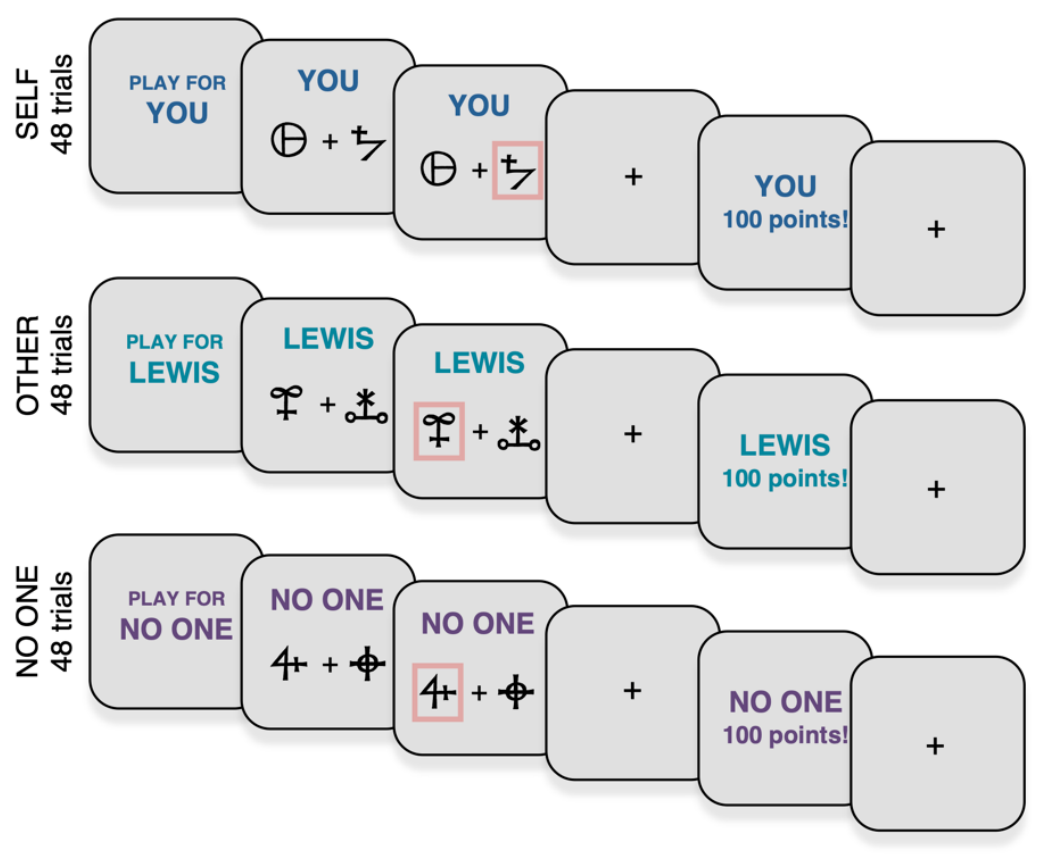

146 Figure 1. Prosocial learning task and social role assignment. (a) The role assignment

147 procedure involved the participant (dark blue), confederate (light blue) and two experimenters

148 (green). Top: from above showing the positioning of the participant and two experimenters

149 inside the testing room, and the confederate the other side of the door. Bottom: the participant

150 and confederate wore a glove to disguise their identity and waved to each other from either

151 side of the door. Participants were instructed that they would be assigned to roles of 'Player

1521 1' and 'Player 2' but the participant was always assigned to be Player 1. After this procedure

153 participants were informed that they would play a game where they could gain rewards for

154 themselves, the other participant (Player 2) or neither participant. They were told that Player

1552 would not play the same game for them and that Player 2 would not know that they may

156 receive an additional bonus based on the choices the participants. This meant that

157 participants' choices were made anonymously and should not be affected by reputational

158 concerns. (b) Participants performed a reinforcement learning task ('prosocial learning task')

159 in which they had to learn the probability that abstract symbols were rewarded to gain points.

160 At the beginning of each block, participants were told who they were playing for, either 161 themselves, for the other participant, or in a condition where no one received the outcome.

162 Points from the 'self' condition were converted into additional payment for the participant 163 themselves, points from the 'other' condition were converted into money for Player 2 and 164 points from the 'no one' condition were displayed but not converted into any money for anyone. 


\section{Results}

We analysed the behaviour of 75 younger adults and 77 older adults who completed the probabilistic reinforcement learning task (Figure 1b), neuropsychological tests, and a measure of psychopathic traits (see Methods). To ensure comparability, older adults with dementia, as diagnosed by the Addenbrooke's Cognitive Examination (ACE) ${ }^{49}$, were not included in the study. The two age groups were matched on gender $\left(X^{2}(1)=0.45, p=0.5\right)$ and did not differ in years of education or IQ (Supplementary Table 1). IQ was quantified using age-standardised scores on the Wechsler Test of Adult

174 Reading (WTAR) ${ }^{50}$. We conducted additional analyses controlling for IQ (standardised 175 WTAR score, measured for young and older adults), and memory and attention (memory and attention subscales of the ACE, older adults only). These control analyses showed that our results are not accounted for by general intelligence or executive function (see Methods and Supplementary Information).

179

\section{Learning occurs for all recipients for both age groups}

We first examined whether participants were able to learn for all three recipients to validate their ability to complete the task. We quantified performance as selecting the option associated with a high chance of receiving reward. Participants in both age groups were able to learn to obtain rewards for themselves, another person, and no one. This was demonstrated through average performance above chance level (50\%; all $t s>15$, all $p s<0.001)$ and a significant effect of trial number in predicting trial-by-trial performance (all $z s>4.48, p s<0.001$ ) for each separate recipient and age group combination.

190

\section{Learning rate depends on who receives reward}

193 Next, to quantify learning, we used computational models of reinforcement learning to estimate learning rates $(\alpha)$ and temperature parameters $(\beta)$, key indices for the speed by which people update their estimates of reward, and the precision with which they make choices, respectively. Models were fitted using a hierarchical approach and 
compared using Bayesian model selection as used previously by Huys et al. ${ }^{51}$ and Wittmann et al. ${ }^{52}$ (see Methods). We tested multiple models that varied with respect to whether learning could be explained by shared or separate free parameters across recipient (self, other, no one). Based on our previous results ${ }^{7}$, we examined whether shared or separate learning rates in particular resulted in a better model fit. We used four candidate models:

203

204 (i) $1 \alpha 1 \beta$ : one $\alpha$ for all three recipients \& one $\beta$ for all three recipients;

205

(ii) $3 \alpha 1 \beta$ : $\alpha$ self, $\alpha$ other $\&$ ano one, one $\beta$;

206

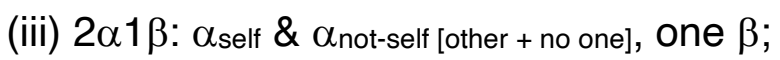

207

(iv) $3 \alpha 3 \beta$ : $\alpha_{\text {self, }} \alpha_{\text {other, }} \alpha_{\text {no one }}, \beta_{\text {self, }} \beta_{\text {other }} \& \beta_{\text {no one }}$ (see Supplementary Table 2 ).

208

Initially, we aimed to establish that both our experimental schedule and our models were constructed in a way that allowed us to disentangle recipient-specific learning rates. To this end, we created synthetic choices using simulations based on each of our four models (see Methods). We fitted the models to the data and assured that the

213 best fitting model was the one that had been used to create the data. In such a way, 214 we established model identifiability, both when considering the exceedance probability 215 (Figure 2a and see Methods) and the number of times a model was identified as the 216 best one (Figure $2 b$ ). As a second prerequisite for testing for agent-specific learning rates, we performed parameter recovery using our key model of interest, the $3 \alpha 1 \beta$ model. Over a wide parameter space, we were able to recover the parameters underlying our choice simulation (Figure 2c).

Having established the models were identifiable and parameters recoverable, we performed Bayesian model selection on the data from our participants. Participant's choices were best characterised by the $3 \alpha 1 \beta$ model. This indicated that the learning process underlying the choices is most accurately captured by assuming separate learning rates for each recipient ( $\alpha_{\text {self, }}$, oother $\& \alpha_{\text {no one). }}$ This model fit the data best (exceedance probability $=97 \% ; \Delta B I C_{i n t}=122$; Figure $2 \mathrm{~d}$ ) and predicted choices well $227 \quad\left(R^{2}=51 \%\right.$; see Methods for further details). 
Higher learning rates are associated with better performance

231 As a final check of the robustness of our model and to enable clear interpretation of

232 any differences in learning rate between recipients and age groups (c.f. ${ }^{53}$ ), we

233 conducted an additional simulation experiment. We simulated data from 10,000

234 participants using the $3 \alpha 1 \beta$ model. This created 30,000 values of $\alpha$ from the three

235 recipient conditions, spanning the full range of possible values from 0 to 1 (see

236 Methods). For each, we quantified the associated performance as the percentage of

237 times the synthetic participant chose the high probability of reward option, averaged

238 across the blocks for the relevant recipient. Plotting the learning rates against

239 performance (Figure 2e) shows that the optimal value of $\alpha$ is approximately 0.55 .

240 This is higher than all the values of $\alpha$ found on our task in any recipient condition for

241 either age group. Therefore, higher learning rates were associated with better

242 performance. We further established this link by correlating learning rates and

243 performance in the empirical data from our participants. We found a strong

244 correlation between learning rates and performance overall $\left(r_{\mathrm{s}(150)}[95 \%\right.$ confidence

245 interval] $=0.58[0.46,0.68], p<0.001$; Figure 2f) and in each recipient and age group

246 combination (Supplementary Table 3). 

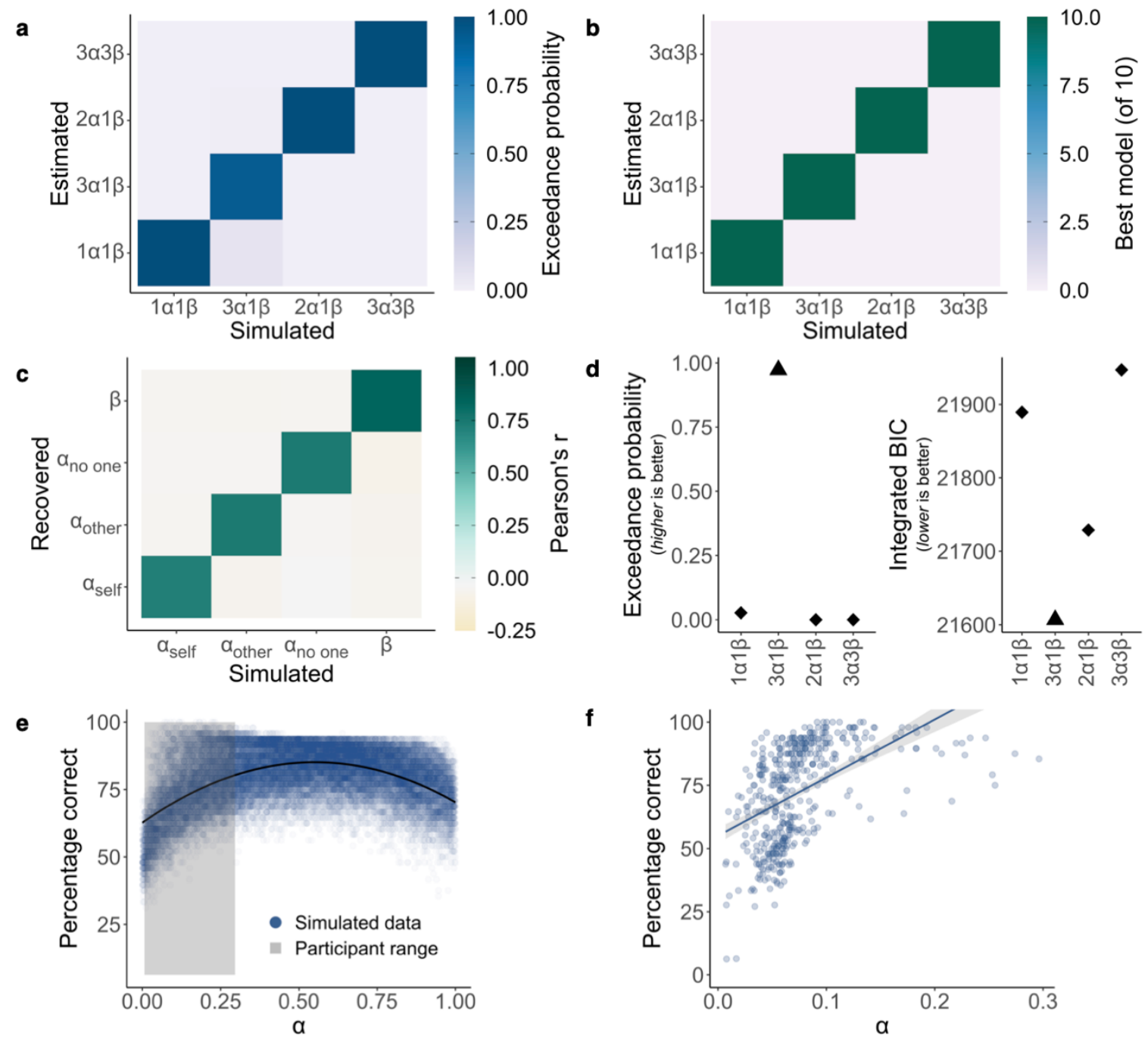

f

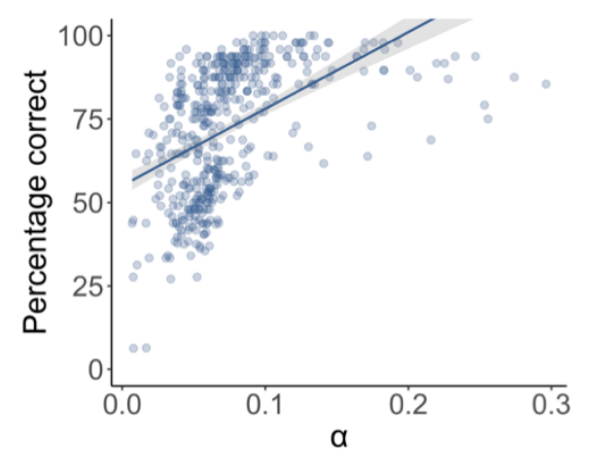

248 Figure 2. Model identifiability, parameter recovery and simulation. (a) Model

249 identifiability average exceedance probability confusion matrix and (b) model identifiability best model selection confusion matrix. Data were simulated from 150 synthetic participants with each of our four models then Bayesian model selection was applied, and this procedure was repeated 10 times. Identifiability is shown by strong diagonals. (c) Parameter recovery was performed on data simulated by the winning $3 \alpha 1 \beta$ model from 1296 synthetic participants. Confusion matrix represents correlations between simulated and fitted parameters. Strong correlations on the diagonal show parameters can be recovered. (d) The $3 \alpha 1 \beta$ model $(\boldsymbol{\Lambda})$ is the best model on both exceedance probability and integrated Bayesian Information Criterion (BIC) fit measures. (e) Average percentages of correct choices (high probability of reward option) associated with 30,000 simulated $\alpha$ values (10,000 synthetic participants, 3 recipient conditions) show that an optimal learning rate is approximately 0.55 in this task. The range of $\alpha$ values for our participants was below this peak (grey shading), such that a higher learning rate was associated with better performance. (f) Correlation between percentage correct and learning rate across participants. There was a significant correlation between learning rate and accuracy $\left(r_{s(150)}=0.58[0.46,0.68], p<0.001\right)$ (see Supplementary Table 3 for each separate age group and recipient combination; $r_{\mathrm{s}}$ in all cases $>0.46, p s<0.001)$. Shaded area represents $95 \%$ confidence interval. 

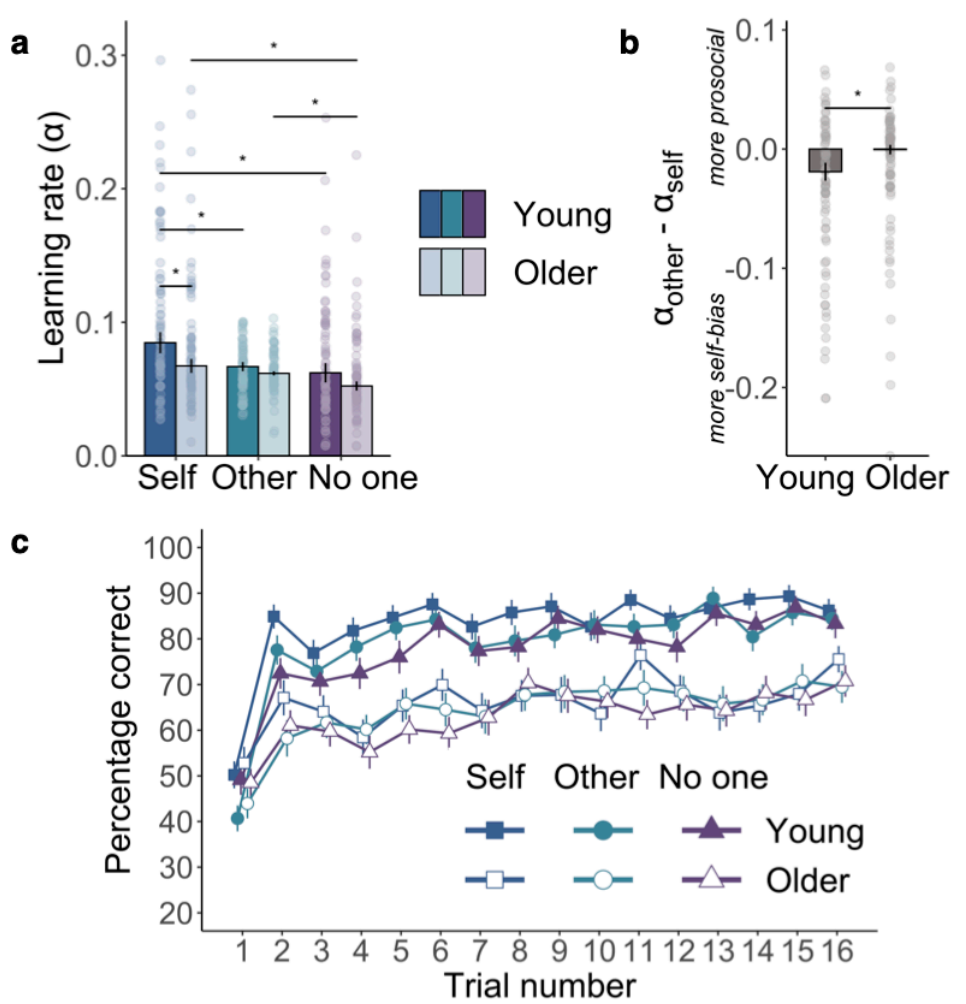

Figure 3. Age-group differences in accuracy and learning rates. (a) Comparison of learning rates from the computational model show older adult's prosocial learning was preserved: learning rates in the other condition did not differ from the self condition or from young adult's prosocial learning rates. Only young participants showed self-bias, $n=150$ (75 young, 75 older). Bars show group median, error bars are standard error of the median, asterisks represent significant between-group and within-group Wilcoxon t-tests $(p<0.05)$. (b) Median difference between learning rates in the other and self conditions illustrates the larger self-bias in young, compared to older, adults, $n=150$ (75 young, 75 older). Error bars are standard error of the median, the asterisk represents the significant age group * recipient [self vs. other] interaction from the robust linear mixed-effects model ( $p<0.001)$. (c) Group-level learning curves showing choice behaviour in the three recipient conditions for each age group. Trials are averaged over the three blocks ( 48 trials total per recipient presented in three blocks of 16 trials) for the self, other, and no one recipients, $n=152$ ( 75 young, 77 older). Points show group mean, error bars are standard error of the mean. 


\section{Older adults show a reduced self-bias in learning rates}

Next, we used this validated computational model to test our hypotheses as to whether there were group differences in learning rates when learning to reward self, other or no one. Two participants had learning rates for two of the three recipients more than three standard deviations (SDs) above the mean ( $\alpha_{\text {self }} 6.68 \& \alpha_{\text {no one }} 9.64 ; \alpha_{\text {self }} 7.96 \&$ dother 3.78 SDs above the mean) and were excluded from all analysis of learning rates.

We analysed the condition-specific learning rates from our best fitting computational model using a robust linear mixed-effects model (RLMM; see Methods). The RLMM fixed effects were age group (young, older), recipient (self, other, no one), as well as the age group * recipient interaction. While this RLMM includes all three recipient conditions, it generates coefficients (main effect and the interaction with age group) contrasting pairs of recipient conditions - [self vs. other] and [self vs. no one]. These are more interpretable than an omnibus test that would not show which recipient conditions were driving an effect or interaction. We followed up these results with planned comparisons, between the older and younger group in each recipient condition, and between pairs of recipient conditions within each age group.

Across age groups, participants showed a higher learning rate when rewards were for themselves, compared to for another person (recipient [self vs. other]: $b=-0.024$ [$0.034,-0.014], z=-4.79, p<0.001)$. Importantly however, this pattern differed between age groups. The difference between learning rates for self and other was reduced in older compared to younger adults (recipient [self vs. other] * age group interaction: $b=0.016[0.002,0.030], z=2.29, p=0.02)$. Between-group comparisons showed older adults learnt more slowly for themselves compared to younger adults $(W=3512, Z=-$ 2.63, $\left.r_{(150)}=0.22[0.06,0.36], p=0.009\right)$. However, prosocial learning was preserved, with a Bayes factor suggesting strong evidence of no difference in $\alpha_{\text {other }}$ between young and older adults $\left(\mathrm{W}=3042, \mathrm{Z}=-0.86, \mathrm{r}_{(150)}=0.07[0.00,0.24], p=0.39, \mathrm{BF}_{01}=4.26\right)$. adults had higher learning rates for themselves, relative to another person $(\mathrm{V}=659$,

$311 Z=-4.04, \quad r(75)=0.47[0.26,0.63], p<0.001)$. In contrast, older adults showed no 
312 significant difference between learning rates for self and other $(V=1150, Z=-1.45$, $\left.r_{(75)}=0.17[0.01,0.38], p=0.15, B_{01}=1.08\right)$.

315 As expected, across age groups learning was slower for no one than self (recipient 316 [self vs. no one]: $b=-0.023$ [-0.033, -0.013], $z=-4.57, p<0.001)$. Unlike $\alpha_{\text {self }}$ vs. $\alpha_{\text {other, }}$ 317 learning for self compared to no one did not interact with age group (recipient [self vs. 318 no one] * age group interaction: $b=0.008$ [-0.006, 0.022], $z=1.15, p=0.25)$. Within-

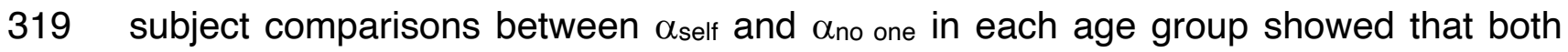
320 groups learnt preferentially for themselves compared to no one (young adults: V=928, $321 Z=-2.62, r_{(75)}=0.30[0.07,0.51], p=0.009$; older adults $\mathrm{V}=901, \mathrm{Z}=-2.76, \mathrm{r}_{(75)}=0.32[0.09$, 322 0.53], $p=0.006)$. There was no significant difference between the age groups in $\alpha_{\text {no one }}$ but also no evidence in support of the null $\left(W=3241, Z=-1.61, r_{(150)}=0.13[0.01,0.29]\right.$, $\left.p=0.11, \mathrm{BF}_{01}=2.04\right)$.

325

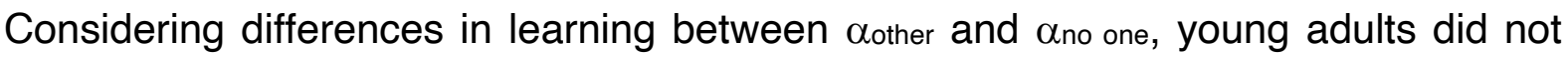
differentiate between another person and no one, with strong Bayesian evidence for no difference $\left(\mathrm{V}=1533, \mathrm{Z}=-0.57, \mathrm{r}_{(75)}=0.07\right.$ [0.00, 0.31], $\left.p=0.57, \mathrm{BF}_{01}=5.08\right)$. In contrast, older adults had higher learning rates for another person, compared to no one $\left(\mathrm{V}=976, \mathrm{Z}=-2.37, \mathrm{r}_{(75)}=0.27\right.$ [0.05, 0.49], $\left.p=0.02\right)$. Crucially, this shows that older adults' lack of differentiation between self and other was not simply because they were insensitive to the recipient condition.

Finally, we also observed an effect of age on both learning rates overall and temperature parameters. Older adults showed slower learning overall compared to younger adults $(b=-0.019[-0.028,-0.009], Z=-3.73, p<0.001)$ and higher levels of exploration of choice options (median $\beta$ young: 0.05 , older: $0.19, W=1511, Z=-4.89$, $r_{(150)}=0.40[0.26,0.53], p<0.001$; Supplementary Figure 1).

340 In summary, older adults prosocial learning was preserved at the same rate as young 341 adults, despite age-related declines in self-relevant learning rates. In other words, young adults showed a self-bias in learning, but older adults distinguished between 
themselves and others significantly less than the young participants. Only older adults, not young adults, distinguished between rewards for another person and no one.

Participants perform better for themselves, compared to no one

348 For completeness, we also tested the effects of recipient and age group on trial-by349 trial tendency to pick the high reward stimuli (Figure 3c). In addition to the main effect of trial number $(b=1.71$ [1.29, 2.13], $z=7.97, p<0.001)$, showing learning, these models revealed older adults chose the high reward option less frequently (mean for young: $80 \%$, older: $64 \%, b=-1.18[-1.65,-0.70], z=-4.84, p<0.001)$, and improved less during the task (trial number * age group interaction $b=-0.81[-1.34,-0.27], z=-2.95, p=0.003$ ) across recipients. When averaging across age groups, performance was better for the self $(75 \%)$, compared to no one $(70 \% ; b=-0.36[-0.05,0.16], z=-2.28, p=0.02)$. However, there was not a significant difference between accuracy for other (72\%) and self $(b=-0.22[-0.49,0.05], z=-1.63, p=0.10)$, or any significant interactions between age group and recipient (bs<0.16, $z s<0.96$, ps $>0.34$ ).

Psychopathic traits are lower in older adults and explain variance in prosocial 362 learning

Finally, we examined individual variability in psychopathic traits, considering agerelated differences and influence on prosocial learning. Several studies have suggested that individual differences in psychopathic traits can be meaningfully and accurately captured in community samples and often parallel findings in criminal offenders ${ }^{43}$. Critically, psychopathic traits are closely linked to alterations in social behaviour and willingness to help others. Therefore, we also asked participants to complete the Self-Report Psychopathy Scale (SRP-IV-SF) ${ }^{39}$. The SRP is a measure of psychopathic traits in healthy samples that assesses traits linked to clinical psychopathy, such as antisociality and interpersonal affect. The measure robustly captures the latent structure of clinical psychopathy to enable parallels can be drawn between normal individual differences in the community and clinical samples (see Methods). One participant in each age group had missing questionnaire data and are 
not included in these analyses. Psychopathic traits are consistently divided into two components that this scale measures: core affective-interpersonal traits, which

377 capture lack of empathy and guilt; and lifestyle-antisocial traits, which capture 378 impulsivity and antisocial tendencies. Comparing the two age groups on these scales 379 showed that older participants had significantly lower scores than young participants 380 on both the core affective-interpersonal (young mean=24.36, older mean=21.09, $\left.\mathrm{W}=3558, \mathrm{Z}=-3.15, \mathrm{r}_{(148)=0.26}[0.11,0.40], p=0.002\right)$ and the lifestyle-antisocial subscales (young mean=22.89, older mean=20.27, $W=3471, Z=-2.82, r_{(148)}=0.23$ $[0.09,0.38], p=0.005)$. These findings suggest that both components of psychopathic traits were reduced in older, compared to younger, adults.

Next, we sought to test our hypothesis that individual differences in core psychopathic traits would explain variability in learning rates, specifically for prosocial learning. We observed a significant negative relationship between $\alpha_{\text {other }}$ and core psychopathic traits among older participants $\left(r_{s(74)}=-0.33[-0.52,-0.11], p=0.005\right.$; Figure $\left.4 a\right)$. Intriguingly, this relationship was significantly more negative $(Z=3.28, p=0.001)$ than the equivalent correlation in young adults, which was not significant $\left(r_{s}(74)=0.21[-0.02\right.$, 392 0.42], $p=0.07$; Figure $4 \mathrm{~b}$ ). This pattern of results was the same when correlating 'relative prosocial learning rate' (the difference between oother - $\alpha_{\text {self }}$ ) with core psychopathic traits. We also conducted control analyses, correlating the same pairs of variables but using partial correlations controlling for $\beta$. The negative relationship between prosocial learning (when quantified as $\alpha_{\text {other }}$ or $\alpha_{\text {other }}-\alpha_{\text {self }}$ ) and psychopathic traits was still present for older adults, showing that the correlations with learning rates were independent of individual choice exploration (all $p s<0.05$; see Supplementary Table 4). The negative relationship between $\alpha_{o t h e r}$ and core psychopathic traits for older adults also remained significant after applying false discovery rate (FDR) correction for multiple comparisons across this correlation and the five other age group $\&$ recipient combinations (see Supplementary Table 5). Moreover, the finding that no significant correlations were apparent between psychopathic traits and $\alpha_{\text {self }}$ or $\alpha_{\text {no one }}$ (ps>0.15; Supplementary Table 5) suggests a specificity in the relevance of psychopathic traits to prosocial learning in older adults. 
407 Given the age-group differences in levels of psychopathic traits and their correlations 408 with prosocial learning, our final analysis considered whether scores on the core 409 affective-interpersonal psychopathic traits measure mediated the effect of age group 410 on relative prosocial learning rate ( $\alpha_{\text {other }}-\alpha_{\text {self }}$ ). A standard mediation model (Figure 4115 a) did not show evidence for a significant mediation. However, as would be predicted 412 if the link between psychopathic traits and prosocial learning depends on age, we 413 found evidence for a moderated mediation. This revealed core psychopathic traits 414 mediated the effect of age group on relative prosocial learning rate for older adults 415 (unstandardised indirect effect $=0.006[0.001,0.013], p=0.008$, proportion 416 mediated=0.32) but not for young adults (unstandardised indirect effect=-0.001 [$4170.007,0.006$ ], $p=0.65$; see Figure $5 \mathrm{~b}$ for standardised coefficients). In summary, young 418 and older adults differed in levels of psychopathic traits and whether or not 419 psychopathic trait scores explained the extent to which their learning rates were 420 relatively prosocial or self-biased. 

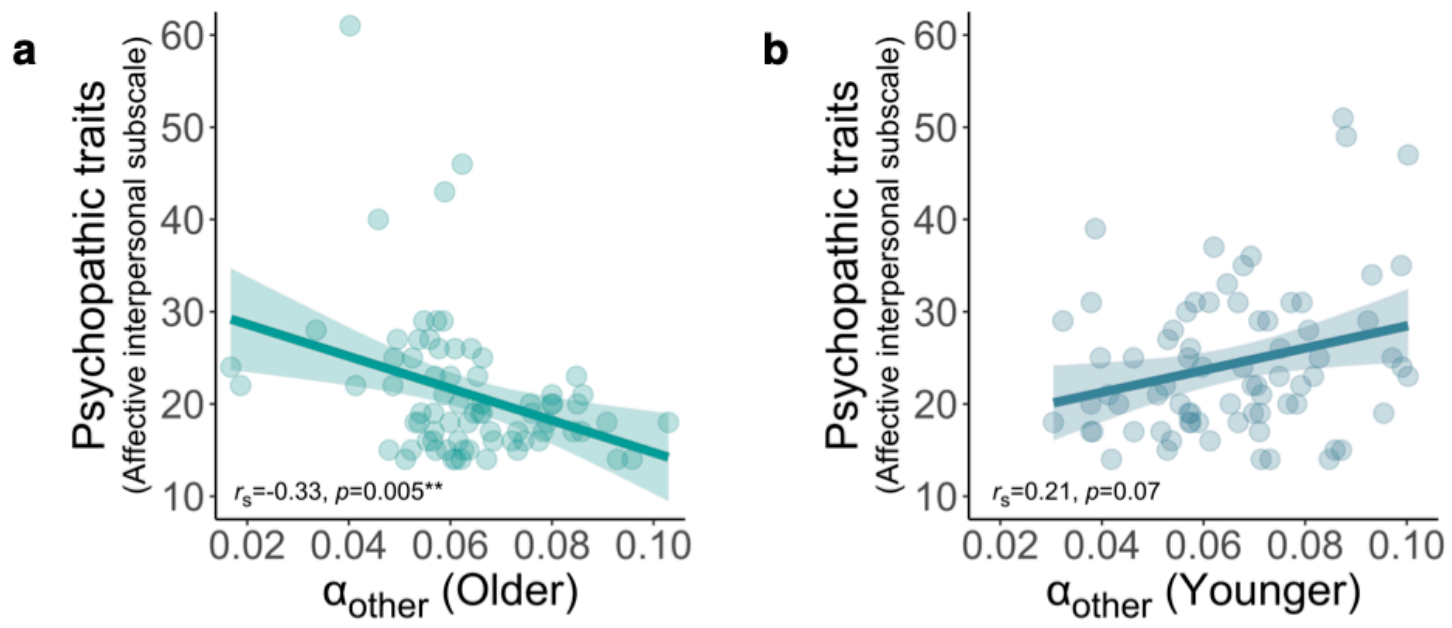

422 Figure 4. Correlations between prosocial learning rates $\left(\alpha_{\text {other }}\right)$ and scores on the 423 affective-interpersonal subscale of the Self-Report Psychopathy scale. (a) For older 424 adults, levels of psychopathic traits are negatively correlated with prosocial learning rates $\left(r_{\mathrm{s}}=-\right.$ 4250.33 [-0.52, -0.11$], p=0.005$, false discovery rate (FDR) corrected $p=0.03$ ). (b) There is no 426 significant relationship for young adults $\left(r_{s}=0.21[-0.02,0.42], p=0.07\right.$, FDR-corrected $\left.p=0.22\right)$ and the correlation is significantly more negative $(Z=3.28, p=0.001)$ for older than young adults. This pattern of results is the same when considering correlations between psychopathic traits and the lack of self-bias in learning ( $\alpha_{\text {other }}-\alpha_{\text {self }}$; not shown). This measure of prosocial learning is also negatively correlated with psychopathic traits in older adults $\left(r_{\mathrm{s}}=-\right.$ $0.25[-0.45,-0.02], p=0.03)$ but not younger adults $\left(r_{s}=0.11[-0.12,0.33], p=0.36\right.$; difference $\mathrm{Z}=2.15, p=0.03)$. Age-group differences in psychopathic traits and the correlation between $\alpha_{o t h e r}$ and psychopathic traits, for older adults only, also remained significant when excluding extreme scores (>3 SDs from the mean) on the psychopathic traits measure (Supplementary Tables $6 \& 7$ ). Shaded areas represent 95\% confidence intervals. 
a

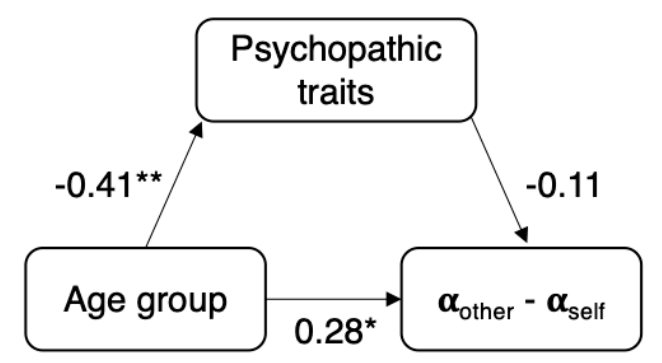

$(0.23)$ b

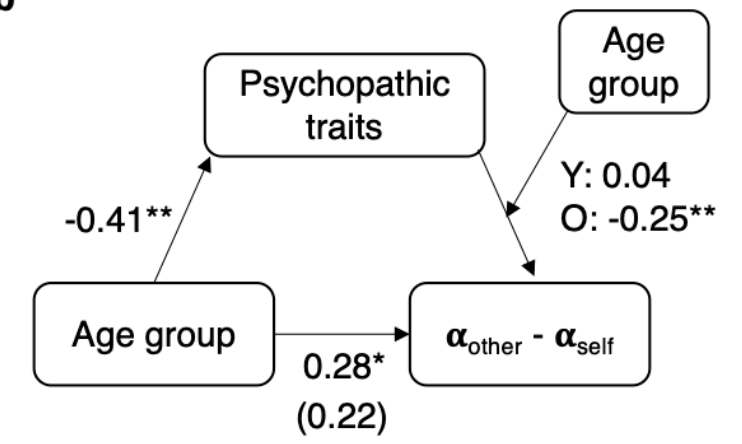

437 Figure 5. Psychopathic traits mediate the effect of age group on relative prosocial

438 learning ( $\left.\alpha_{\text {other }}-\alpha_{\text {self }}\right)$ for older adults only. (a) A standard mediation model does not show 439 evidence that psychopathic traits mediate the effect of age group on relative prosocial

440 learning. Although accounting for psychopathic traits means the significant direct effect of 441 age group on relative prosocial learning (standardised coefficient $=0.28, p=0.04$ ) becomes 442 non-significant (standardised coefficient $=0.23, p=0.11$ ), psychopathic traits do not predict 443 relative prosocial learning overall so there is no mediation. (b) Evidence of a moderated 444 mediation is revealed when accounting for differences between young and older adults in 445 how psychopathic traits predict relative prosocial learning. Psychopathic traits are a mediator 446 for older but not younger adults. Psychopathic traits are scores on the affective-interpersonal 447 subscale of the Self-Report Psychopathy scale, Y: young, O: older, asterisks represent 448 significant effects $\left({ }^{*} p<0.05,{ }^{\star *} p<0.01\right)$. 


\section{Discussion}

Reinforcement learning is a fundamental process for adaptive behaviour in many species. However, existing studies have largely focused on young people and selfrelevant learning, but the decisions we make often occur in a social context 54 and our actions affect outcomes for others. Here, for the first time, we applied computational models of reinforcement learning to the question of ageing-related changes in selfrelevant and prosocial learning. We found a clear decrease in learning rates for selfrelevant rewards in older, compared to younger, adults. Intriguingly, despite this reduction in self-relevant learning, learning rates for outcomes that affected others did not differ between older and young adults, with Bayesian analyses supporting no rates, age was also associated with lower psychopathic traits, which were specifically linked to prosocial learning ability in older adults.

463

Models of learning are a powerful tool for understanding prosocial behaviour. By isolating the learning rate, we can precisely examine the influence of reward history on learning. We robustly replicated previous findings that self-relevant learning can be computationally separated from prosocial learning ${ }^{7}$, with different learning rates for different recipients providing the best explanation of behaviour. Including separate learning rates improved the model fit and, on average across participants, there was a self-bias, learning rates were higher for self-relevant rewards, compared to when someone else received the reward. However, this self-bias was reduced in older adults who showed higher prosocial learning rates, relative to their own self-relevant learning rates, than young adults. As expected, older adults learnt more slowly for themselves

474 than young adults but in the prosocial condition, the learning rates did not significantly 475 differ between the age groups. Bayesian analyses additionally confirmed that prosocial learning was preserved between young and older adults.

478 As with much research on age-related changes on cognitive and social tasks, our key 479 finding that self-bias in learning is reduced in older adults could be interpreted as due 480 to changes in ability, motivation, or a combination of these factors. Importantly, 
learning rates were not associated with executive function or an age-standardised measure of intelligence. We also show that our results remain the same after controlling for these measures. These findings suggest that the observed decline in self-relevant learning rates, but preservation of prosocial learning rates, for older adults are not explained by changes in these broad abilities. Considering learning more specifically, a recent comparison of motivation and ability as explanations for ageing-related reductions in model-based strategies during self-relevant learning supported the limited cognitive abilities account ${ }^{55}$. Our finding that learning rates for self-relevant outcomes were reduced in older adults is in line with a degeneration in the neurocognitive systems required for successful learning. Research combining models of learning with neuroimaging and pharmacological manipulations suggests ageing reduces the ability to generate reward prediction errors ${ }^{18}$ (but see ${ }^{56,57}$ ) due to declines in dopamine functioning 28,58 (also see ${ }^{59,60}$ ). Differences in motivation could also be applicable for self-relevant learning as the subjective value of financial outcomes is also likely to decrease in older age, due to changes in wealth across the lifespan ${ }^{34}$.

Our findings suggest that despite declines in learning ability associated with ageing, prosocial learning - learning to help others - is preserved. This finding aligns with an emerging literature showing older adults may be more prosocial and less self-biased than younger adults $29,30,61$. The possibility that relatively preserved prosocial learning is related to increased prosocial motivation is in-line with our observed link between learning rates and psychopathic traits. Psychopathic traits were significantly reduced in our older adult sample, dovetailing with similar previous findings on this trait 47,48 and broader trait benevolence ${ }^{33}$. Importantly, we found psychopathic traits in older adults negatively correlated with prosocial learning rates. Self-bias in learning rates (i.e. higher learning rate for self compared to other) was most reduced, and even reversed, in the older people lowest on psychopathic traits. Notably, this negative correlation 509 between psychopathic traits and prosocial learning was only found for older adults.

510 This suggests that age-related differences in prosocial learning could be linked to 511 basic shifts in individual traits and motivations over the lifespan, not just to domain512 general reductions in cognitive abilities. The idea that social motivations become more 
513 influential in learning and decision-making with age has also been suggested based

514 on studies of social rewards such as smiling faces or hypothetical time spent with 515 social partners ${ }^{62-64}$. Taken together, this work suggests that strategies to support 516 healthy ageing might benefit from leveraging potentially preserved social motivations.

518 Importantly, our task included a control condition where no one benefitted. This was 519 essential to establish that the lack of difference between self and other learning rates 520 for older adults was not simply due to an age-related reduction in the absolute dynamic range as maximum learning rates decrease. Older adults had higher learning rates for

522 both others and themselves, compared to this control condition. In contrast, young 523 adults did not differentiate another person from no one. Older adults therefore showed a relative increase in learning rates that was specific to the prosocial condition. This is also evidence against the idea that lower learning rates in older people are reflective of a general reduced sensitivity to who gets the reward. It is interesting to note that the magnitude of the decrease in self-relevant learning rates associated with being older, compared to younger, is similar to the decrease associated with a young person learning for someone else, compared to themselves. The preservation of prosocial learning rates between age groups may seem at odds with the decreased self-relevant learning rates in our sample of older adults and existing evidence of underlying neurobiological deterioration. However, learning from outcomes for self and other have been linked to distinct regions of the brain in humans, shown though neuroimaging 7,65 , and causally in monkeys with focal lesions ${ }^{66}$.

Taken together, our results add to a growing body of literature suggesting age-related increases in prosocial motivation. If this is the case, the next question is how and why this happens, as there are many possible reasons to be prosocial. For example, prosocial behaviours can be motivated by reputational concerns ${ }^{67}$, the 'warm glow' of 540 helping ${ }^{68-70}$, or vicarious reinforcement from positive outcomes for others ${ }^{71}$. In our 541 procedure, we were very careful to prevent reputational concerns influencing learning 542 to help others. Participants underwent an extensive procedure to introduce them to 543 another participant but to hide information about their age and identity. This meant we 544 could assess tendency for prosocial learning in a situation where reputational 
545 motivations were excluded, and identity-based influences were controlled. Using a

546 reinforcement learning task, in which performance generates positive outcomes for

547 others also focusses on vicarious rewards from the outcome, rather than warm glow

548 associated with the action of helping. Thus, the increase in prosocial learning rates, 549 relative to self-relevant learning rates, suggests older adults are reinforced by 550 outcomes for others and themselves more similarly than younger adults. Many 551 prosocial measures such as the dictator game ${ }^{72}$ are costly, requiring direct trade-offs 552 between outcomes for oneself and others. This also makes it hard to detect whether 553 changes are in the value of outcomes for oneself, or outcomes for others, or both. In 554 contrast, separating self-relevant learning, prosocial learning, and the control condition 555 allows us to differentiate increases in the value of prosocial outcomes from decreases 556 in the value of outcomes for the self. Our results are consistent with older adults having 557 both decreased self-relevant learning rates (compared to young adults) and increased 558 prosocial learning rates (compared to their performance in the control condition).

560 Our results also support the advantages of a model-based approach for understanding both prosocial behaviour and ageing. The model-based parameters were more sensitive to the effects of interest than general measures of performance on the task. The model comparison process is able to provide important information about how the learning process takes place, which cannot be revealed from performance measures alone. We showed that learning was best represented by a separate learning rate for each recipient. Moreover, this approach is able to capture additional latent parameters that drive behaviour, such as the inverse temperature, which indexes how closely participants follow the stimulus value. We demonstrate that a single inverse temperature parameter best explained behaviour during learning across recipient conditions, despite learning rates being distinct.

572 While our procedure and task have many benefits, it is important to also recognise 573 limitations. To test for age-related differences in prosocial learning, we recruited a 574 group of older adults and a group of younger adults. This increases power to detect 575 differences, but we are unable to assess at what age or how quickly changes take 576 place. Also, while our age groups were matched on years of education and IQ, the 
07

recruitment from university databases or issues around self-selection may mean that the levels of education and IQ in our sample are not completely representative of the general population. Further studies could include samples recruited entirely from the community and participants across the whole adult lifespan. Future studies should also assess the timescale after which older participants reach similar ceiling levels of performance as younger adults, or whether they are never able to reach the same level of performance. Of similar importance is the question of whether older adults may be able to sustain higher levels of motivation over an extended period of time compared to younger adults, possibly compensating for slower learning speeds. In this study, due to time constraints and the presence of several conditions, we are only able to derive conclusions from a limited time window. Moreover, previous research has suggested that individual differences in empathy - the ability to vicariously experience and understand others' affect - might relate to differences in prosocial learning ${ }^{7}$. Empathy is positively associated with affective-interpersonal psychopathic traits and might also relate to motivation to help others ${ }^{73,74}$. Further studies could also assess how empathy predicts changes in prosocial learning across the lifespan. Finally, future studies could manipulate the identity of the recipient as we show a preservation in tendency to help others when there are no particular characteristics known about the other person, but these effects might additionally be modulated by factors such as perceived social distance.

To conclude, we find new evidence that despite declines in self-relevant learning in older adults, the ability to learn which actions benefit others is preserved. Moreover, the bias with which people favour self-relevant outcomes is reduced. Not only do older adults have relatively preserved prosocial learning, they also report lower levels of core psychopathic traits that index lack of empathy and guilt, and this trait difference is linked to the changes in prosocial learning. These findings could have important implications for our understanding of reinforcement learning and theoretical accounts of healthy ageing. 
Materials and Methods

609

\section{Participants}

611

612 We recruited 80 young participants and 80 older participants using the same 613 recruitment methods in order to match the samples as closely as possible. Participants 614 were recruited from university databases, which included students and members of 615 the community, social media, and adverts in local newspapers. We excluded anyone 616 who was currently studying or had previously studied psychology and no one took part 617 for course credit. Additional exclusion criteria were previous or current neurological or 618 psychiatric disorder, non-normal or non-corrected to normal vision and, for the older 619 sample, scores on the Addenbrooke's Cognitive Examination that indicate potential 620 dementia (cut-off score 82 ) ${ }^{49}$. This sample size gave us $88 \%$ power to detect a medium 621 size effect $(d=0.5)$.

622

623 Five young and three older participants were excluded due to: diagnosis of a 624 psychiatric disorder at the time of testing (1 young participant); previous study of 625 psychology (2 young participants); and incomplete or low-quality data (2 young and 3 older participants). This left a final sample of 152 participants, 75 young adults (44 627 females aged 18-36, mean=23.07) and 77 older adults (40 females aged 60-80, mean=69.84). Two older participants were excluded from all analyses involving 629 learning rates due to each having two learning rate estimates more than three standard deviations (SDs) above the mean (for one $\alpha_{\text {self }}$ was 6.68 SDs above the mean \& $\alpha_{\text {no }}$ one 9.64 SDs above the mean; for the second aself was $7.96 \&$ oother 3.78 SDs above the mean). One further participant from each age group was missing data on the SRP measure so are not included in the relevant analyses.

635 Participants were paid at a rate of $£ 10$ per hour plus an additional payment of up to $£ 5$ depending on the number of points they earned for themselves during the task. They were also told the number of points that they earned in the prosocial condition would translate into an additional payment of up to $£ 5$ for the other participant (see details of the task below). All participants provided written informed consent and the study was 
approved by the Oxford University Medical Sciences Inter Divisional Research Ethics

641 Committee and National Health Service Ethics.

642

\section{Prosocial learning task}

644

645 The prosocial learning task is a probabilistic reinforcement learning task, with rewards 646 in one of three recipient conditions: for the participant themselves (self), for another 647 participant (other; prosocial condition), and for no one (control condition). Each trial 648 presents two symbols, one associated with a high (75\%) probability of gaining points 649 and the other with a low (25\%) probability of gaining points. The two symbols were 650 randomly assigned to the left or right side of the screen and selected via a corresponding button press. Participants select a symbol then receive feedback on whether they obtained points or not (see Figure 1b) so learn over time which symbol maximises rewards. The experiment was subdivided into blocks, i.e. 16 trials pairing the same two symbols for the same recipient. Participants completed three blocks, a total of 48 trials, in each recipient condition, resulting in 144 trials overall (see Supplementary Information for trial structure). Blocks for different recipients were pseudo-randomly ordered such that the same recipient block did not occur twice in a row.

660 On trials in the self condition, points translated into increased payment for the participant themselves. These blocks started with "play for you" displayed and had the word "you" at the top of each screen. Blocks in the no one condition had "no one" in place of "you" and points were not converted into any extra payment for anyone. In the prosocial 'other'condition, participants earned points that translated into additional payment for a second participant, actually a confederate. Participants were told that this payment would be given anonymously, they would never meet the other person, and that the person was not even aware of them completing this task (see

668 Supplementary Information). The name of the confederate, gender-matched to the 669 participant, was displayed on these blocks at the start and on each screen (Figure 1b). 670 Thus, participants were explicitly aware who their decisions affected on each trial. 
671 Stimuli were presented using Presentation (Neurobehavioral Systems -

672 https://www.neurobs.com/).

673

\section{Questionnaire measures}

675

676 Dementia screening and executive function. Older adults were screened for 677 dementia using the Addenbrooke's Cognitive Examination (ACE-III) ${ }^{49}$. The ACE 678 examines five cognitive domains; attention, memory, language, fluency and 679 visuospatial abilities. The ACE-III is scored out of 100 and as a screening tool, a cut680 off score of $82 / 100$ denotes significant cognitive impairment. We also used scores on 681 the attention and memory domains in control analyses as proxies for executive 682 function in older adults.

683

684 General intelligence. All participants completed the Wechsler Test of Adult Reading 685 (WTAR $)^{50}$ as a measure of IQ. The WTAR requires participants to pronounce 50 words 686 that have unusual grapheme-to-phoneme translation. This means the test measures reading recognition or existing knowledge of the words, rather than ability to apply rules for pronunciation. The WTAR was developed and standardised with the Wechsler Memory and Adult Intelligence Scales and correlates highly with these measures ${ }^{75}$. Standardisation involves adjusting for healthy age-related differences. The test is suitable for participants aged 16-89, covering our full sample, and scores in older age have been shown to correlate with cognitive ability earlier in life ${ }^{76}$.

693

694 Psychopathic traits. Participants completed the short form of the Self-Report 695 Psychopathy Scale $4^{\text {th }}$ Edition (SRP-IV-SF) ${ }^{39}$. This scale consists of 29 items, 7 each measuring: interpersonal, affective, lifestyle and antisocial tendencies (plus 'I have been convicted of a serious crime'). We used the two-factor grouping, summing the core, affective-interpersonal items and separately, the lifestyle-antisocial items for use

699 in analysis. The robust psychometric properties of this measure have been established 700 in community ${ }^{77}$ and offender populations through construct and convergent validity ${ }^{78}$, 701 internal consistency, and reliability ${ }^{39}$. 
Role assignment. To enhance belief that points earned in the prosocial condition benefitted another person, we conducted a role assignment procedure based on a set-

707 up used in several studies of social decision-making ${ }^{79,80}$. Participants were instructed not to speak and wore a glove to hide their identity. A second experimenter brought the confederate, also wearing a glove, to the other side of the door. Participants only ever saw the gloved hand of the confederate, but they waved to each other to make it

711 clear there was another person there (Figure 1a). The experimenter tossed a coin to 712 determine who picked a ball from the box first and then told the participants which 713 roles they had been assigned to, based on the ball they picked. Our procedure 714 ensured that participants always ended up in the role of the person performing the 715 prosocial learning experiment. Participants were unaware of the age of the other 716 person, but the experimenter used a name for them suggesting their gender was the 717 same as the participant.

718

719 Task procedure. Participants received instructions for the learning task and how the 720 points they earned would be converted into money for themselves and for the other 721 participant. Instructions included that the two symbols were different in how likely it 722 was that choosing them lead to points but that which side they appeared on the screen 723 was irrelevant. Participants then completed one block of practice trials before the main 724 task and were aware outcomes during the practice did not affect payment for anyone. 725 After the task, participants completed the measure of psychopathic traits and the 726 dementia screening 


\section{Computational modelling}

728

729 We modelled learning during the task with a reinforcement learning algorithm ${ }^{16}$, 730 creating variations of the models through the number of parameters used to explain 731 the learning rate and temperature parameters in the task ${ }^{81}$. The basis of the 732 reinforcement learning algorithm is the expectation that an action (or stimulus) $a$ will 733 provide reward on the following trial. This expected value, $Q_{t+1}(a)$ is quantified as a 734 function of current expectations $Q_{t}$ (a) and the prediction error $\delta_{t}$, which is scaled by 735 the learning rate $\alpha$ :

736

737

$$
Q_{t+1}(a)=Q_{t}(a)+\alpha \times \underbrace{\left[r_{t}-Q_{t}(a)\right]}_{\text {Prediction error } \delta_{t}}
$$

738

739

Where $\delta_{t}$, the prediction error, is the difference between the actual reward experienced

740 on the current trial $r_{t}$ ( 1 for reward and 0 for no reward) minus the expected reward on the current trial $Q_{t}(a)$.

742

743 The learning rate $\alpha$ therefore determines the influence of the prediction error. A low 744 learning rate means new information affects expected value to a lesser extent. The softmax link function quantifies the relationship between the expected value of the action $Q_{t}(a)$ and the probability of choosing that action on trial $t$ :

$$
p_{t}\left[\left(a \mid Q_{t}(a)\right)\right]=\frac{e^{\left(Q_{t}(a) / \beta\right)}}{\sum_{a^{\prime}} e^{\left(Q_{t}\left(a^{\prime}\right) / \beta\right)}}
$$

749

750 The temperature parameter $\beta$ represents the noisiness of decisions - whether the 751 participant explores or always chooses the option with the highest expected value. A 752 high value for $\beta$ means choices seem random as they are equally likely irrespective of 753 the expected value of each option. A low $\beta$ leads to choosing the option with the 754 greatest expected value on all trials. 
We used MATLAB 2019b (The MathWorks Inc) for all model fitting and comparison.

760 To fit the variations of the learning model (see below) to (real and simulated) participant data we used an iterative maximum a posteriori (MAP) approach previously described 51,52 . This method provides a better estimation than a single-step maximum likelihood estimation (MLE) alone by being less susceptible to the influence of outliers. It does this via implementing two levels: the lower level of the individual subjects and the higher-level reflecting our full sample. For the real participant data, we fit the model across groups to provide the most conservative comparison, so this full sample combined young and older participants.

768

For the MAP procedure, we initialized group-level Gaussians as uninformative priors with means of 0.1 (plus some added noise) and variance of 100 . During the expectation, we estimated the model parameters ( $\alpha$ and $\beta$ ) for each participant using an MLE approach calculating the log-likelihood of the subject's series of choices given the model. We then computed the maximum posterior probability estimate, given the observed choices and given the prior computed from the group-level Gaussian, and recomputed the Gaussian distribution over parameters during the maximisation step. We repeated expectation and maximization steps iteratively until convergence of the posterior likelihood summed over the group, or a maximum of 800 steps. Convergence was defined as a change in posterior likelihood $<0.001$ from one iteration to the next. Note that bounded free parameters were transformed from the Gaussian space into the native model space via appropriate link functions (e.g. a sigmoid function in the case of the learning rates) to ensure accurate parameter estimation near the bounds. The detailed code for the models and implementation of the fitting algorithm can be found here: https://osf.io/xgw7h/?view_only=bea7b82435b344c5a26f80fd21d8ce19.

\section{Model comparison}

Our hypotheses generated four models to compare that differed in whether the model 
789

790

791

792

793

794

795

796

797

798

799

800

801

802

803

804

805

806

807

808

809

810

811

812

813

814

815

816

817

818

819

820

depended on the recipient (self, other and no one). For model comparison, we calculated the Laplace approximation of the log model evidence (more positive values indicating better model fit ${ }^{82}$ ) and submitted these to a random-effects analysis using the spm_BMS routine 83 from SPM 8 (http://www.fil.ion.ucl.ac.uk/spm/software/spm8/). This generates the exceedance probability: the posterior probability that each model is the most likely of the model set in the population (higher is better, over .95 indicates strong evidence in favour of a model). For the models of real participant data, we also calculated the integrated $\mathrm{BIC}$ (lower is better ${ }^{51,52}$ ) and $\mathrm{R}^{2}$ as additional measures of model fit. To calculate the model $\mathrm{R}^{2}$, we extracted the choice probabilities generated for each participant on each trial from the winning model. We then took the squared median choice probability across participants. The $3 \alpha 1 \beta$ model had the best evidence on all measures (see Supplementary Table 2).

\section{Simulation experiments}

We used simulation experiments to assess that our experiment allowed us to dissociate models of interest, as well as parameters of interest within the winning model. We simulated data from all four models to establish that our model comparison procedure (see above) could accurately identify the best model across a wide range of parameter values. For this model identifiability analysis, we simulated data from 150 participants, drawing parameters from distributions commonly used in the reinforcement learning literature ${ }^{84,85}$. Learning rates $(\alpha)$ were drawn from a beta distribution (betapdf(parameter,1.1,1.1)) and softmax temperature parameters $(\beta)$ from a gamma distribution (gampdf(parameter,1.2,5)). We fitted the models to this simulated data set using the same MAP process as applied to the experimental participants' data and repeated this whole procedure 10 times. By plotting the confusion matrices of average exceedance probability (across the 10 runs; Figure 2a) and how many times each model won (Figure $2 b$ ), we show the models are identifiable using our model comparison process.

Our winning model contained 4 free parameters ( $\left.\alpha_{\text {self }}, \alpha_{\text {other, }} \alpha_{\text {no one }}, \beta\right)$. To assess the reliability of this model and the interpretability of the free parameters, we also 
performed parameter recovery on simulated data (see Supplementary Information for

822 procedure) as recommended for modelling analyses that use a 'data first'

823 approach ${ }^{81,86}$. We simulated choices 1296 times using our experimental schedule and

824 fitted them using MAP. We found strong Pearson's correlations between the true 825 simulated and fitted parameter values (all $r s>0.7$, see Figure 2c), suggesting our 826 experiment was well suited to estimate the model's parameters.

828 Finally, we conducted a principled simulation experiment to identify the optimal 829 learning rate in our task and examine the link between learning rates and performance. 830 We simulated data from 10,000 participants with learning rates $(\alpha)$ and softmax 831 temperature parameters $(\beta)$ drawn from beta and gamma distributions respectively, as 832 described above. We bounded the $\beta$ parameters at 0 and 0.3 to reflect the range 833 shown by our participants. In line with our winning model, simulated participants had 834 a separate learning rate for each recipient condition, and these spanned the full range of possible a values from 0 to 1 . This generated 30,000 learning rate values $(10,000$ participants and three conditions). For each we quantified performance as the

837 proportion of times the simulated participant chose the 'correct' high reward option in 838 the relevant condition. Results showed that average performance improved as 839 learning rates increased, up to learning rates of approximately 0.55 (Figure 2e). 840 Crucially, this optimal alpha was above the highest learning rate shown by any of our 841 participants in any recipient condition, meaning a higher learning rate on our task was associated with better performance.

\section{Statistical analysis}

846 Analysis of group and recipient differences in the fitted model parameters and 847 behavioural data was run in $\mathrm{R}^{87}$ with $\mathrm{R}$ Studio ${ }^{88}$. We used a robust linear mixed-effect 848 model (RLMM; rlmer function; robustlmm package ${ }^{89}$ to predict learning rates and 849 generalised linear mixed-effects models (glmer function; Ime4 package ${ }^{90}$ ) for the trial850 by-trial data (binary outcome of choosing the high vs. low reward option). We used 851 (robust / generalised) linear mixed-effects models as these account for the within852 subject nature of the recipient manipulation and do not rely on parametric 
assumptions. Additionally, unlike an anova model or omnibus test, an RLMM generates coefficients (with confidence intervals and significance values) for terms that compare pairs of factor levels (for example self vs. other). This approach is more informative than an anova when the factor has more than two levels, which is our case as the recipient factor has three levels (self, other, no one). Each linear mixed-effects model had fixed effects of age group, recipient (self, other, no one), and their interaction, plus a random subject-level intercept. Analysis of trial-by-trial choices also included trial number in the fixed terms, interacting with recipient and group (including the three-way interaction), and in the random terms, interacting with recipient. In the analysis of learning rates controlling for IQ, standardised scores on the WTAR were also included as a fixed term (Supplementary Table 8). Correlations of learning rates with psychopathic traits (Supplementary Table 4 \& 5) and neuropsychological measures (Supplementary Table 9) were calculated with Spearman's Rho nonparametric tests. To control for IQ and executive function in the associations between older adults' prosocial learning rates and psychopathic traits, we ran partial correlations each controlling for one of WTAR, ACE memory and ACE attention scores

869 (Supplementary Table 10).

871 For simple and post hoc comparisons, we used two-sided nonparametric tests as 872 outcome variables violated normality assumptions. Effect sizes and confidence 873 intervals for paired and independent nonparametric comparisons were calculated 874 using the cohens_d and wilcox_effsize functions respectively from the rstatix 875 package ${ }^{91}$. Bayes factors $\left(\mathrm{BF}_{01}\right)$ for non-significant results were calculated using 876 nonparametric paired and independent t-tests in JASP92 with the default prior. BF 01 877 corresponds to how many times more likely the data are under the null hypothesis of 878 no difference than under the alternative hypothesis that there is a difference. $\mathrm{A} \mathrm{BF}_{01}$ 879 larger than 3 (equal to $\mathrm{BF}_{10}$ less than 1/3) is considered substantial evidence in favour 880 of the null hypothesis whereas a $\mathrm{BF}_{01}$ between $1 / 3$ and 3 indicates the data cannot 881 clearly differentiate between hypotheses ${ }^{93}$. Median learning rates and their standard errors for plotting were calculated using bootstrapping with 1,000 samples. 
884 For the mediation analysis, we used the mediate function (mediation package ${ }^{94}$ ) 885 combined with robust linear models ( $r$ Im function, MASS package ${ }^{95}$ ). This method 886 estimates the unstandardised indirect effects, with 95\% confidence intervals and 887 significance, through a bootstrapping procedure with 10,000 bootstrapped samples.

888 The outcome in the mediation model was relative prosocial learning rate ( $\alpha_{\text {other }}$ 889 aself), the predictor was age group, and the mediator was psychopathic traits on the 890 core affective-interpersonal subscale of the SRP-IV-SF. We calculated two mediation 891 models. The first, a standard mediation model, included an indirect path from age 892 group to relative prosocial learning rate via psychopathic traits as a mediator (Figure 893 5a). The second included an interaction between age group and psychopathic traits 894 in predicting relative prosocial learning rate to allow the possibility of a moderated 895 mediation $^{96}$. Specifically, this type of moderated mediation examines whether the 896 effect of the mediator (in this case psychopathic traits) on the outcome ( $\alpha_{\text {other }}-\alpha_{\text {self }}$ ) 897 is moderated by the predictor (age group) ${ }^{97,98}$. In other words, psychopathic traits 898 could be a mediator for one age group but not the other (Figure 5b). 


\section{Data availability}

900

901 Data are available at:

902 https://osf.io/xgw7h/?view_only=bea7b82435b344c5a26f80fd21d8ce19.

903

904

\section{Code availability}

905

906 Code for modelling and analysis is available at:

907 https://osf.io/xgw7h/?view_only=bea7b82435b344c5a26f80fd21d8ce19.

908

909

\section{Acknowledgements}

910

911 This work was supported by a Medical Research Council Fellowship (MR/P014097/1), 912 a Christ Church Junior Research Fellowship, and a Christ Church Research Centre 913 Grant to PL; a Wellcome Trust Principal Fellowship to MH; NIHR Biomedical Research 914 Centre, Oxford. The Wellcome Centre for Integrative Neuroimaging is supported by 915 core funding from the Wellcome Trust (203139/Z/16/Z).

916

917 We are grateful to Craig Neumann for assistance with the Self-Report Psychopathy 918 Scale. We are also grateful to the many people who acted as confederates for us 919 during the study.

920

921

\section{Author contributions}

922

923 PL designed the study. AA, LH, DD \& PL collected the data. JC, MW \& PL analysed 924 the data. JC, MW, MH \& PL wrote the paper.

925

\section{Competing interests}

927

928 The authors declare no competing interests 


\section{References}

1. Cohen, M. X. Neurocomputational mechanisms of reinforcement-guided learning in humans: A review. Cogn. Affect. Behav. Neurosci. 8, 113-125 (2008).

2. Glimcher, P. W. Understanding dopamine and reinforcement learning: The dopamine reward prediction error hypothesis. Proc. Natl. Acad. Sci. U. S. A. 108, 15647-15654 (2011).

936

3. Niv, Y. Reinforcement learning in the brain. J. Math. Psychol. 53, 139-154

937 (2009).

938

4. O'Doherty, J. P., Lee, S. W. \& McNamee, D. The structure of reinforcementlearning mechanisms in the human brain. Curr. Opin. Behav. Sci. 1, 94-100 (2015).

5. Schultz, W. Neuronal Reward and Decision Signals: From Theories to Data. Physiol. Rev. 95, 853-951 (2015).

6. Lockwood, P. L. et al. Neural mechanisms for learning self and other ownership. Nat. Commun. 9, 4747 (2018).

7. Lockwood, P. L., Apps, M. A. J., Valton, V., Viding, E. \& Roiser, J. P.

Neurocomputational mechanisms of prosocial learning and links to empathy.

Proc. Natl. Acad. Sci. 113, 201603198-201603198 (2016).

8. Blanchard-Fields, F. Everyday Problem-solving and Emotion: An Adult

9. Blanchard-Fields, F. \& Hess, T. M. The Social Cognitive Perspective and the 
954 10. Samanez-Larkin, G. R. \& Knutson, B. Decision making in the ageing brain: changes in affective and motivational circuits. Nat. Rev. Neurosci. 16, 278-289

956 (2015).

11. Holt-Lunstad, J., Smith, T. B., Baker, M., Harris, T. \& Stephenson, D. Loneliness and social isolation as risk factors for mortality: a meta-analytic review. Perspect. Psychol. Sci. J. Assoc. Psychol. Sci. 10, 227-237 (2015).

960

961

12. Fehr, E. \& Fischbacher, U. The nature of human altruism. Nature 425, 785-791

962 (2003).

13. Buchanan, K. E. \& Bardi, A. Acts of Kindness and Acts of Novelty Affect Life Satisfaction. J. Soc. Psychol. 150, 235-237 (2010).

14. Raposa, E. B., Laws, H. B. \& Ansell, E. B. Prosocial Behavior Mitigates the (2016).

15. Post, S. G. Altruism, Happiness, and Health: It's Good to Be Good. Int. J. Behav. Med. 12, 66-77 (2005).

16. Sutton, R. S. \& Barto, A. G. Reinforcement learning: An introduction. (MIT Press, 2011).

17. Schultz, W. Multiple dopamine functions at different time courses. Annu. Rev. Neurosci. 30, 259-288 (2007).

18. Eppinger, B., Hämmerer, D. \& Li, S.-C. Neuromodulation of reward-based learning and decision making in human aging. Ann. N. Y. Acad. Sci. 1235, 1-17 (2011).

19. Mell, T. et al. Effect of aging on stimulus-reward association learning. 
978

979

980

981

982

983

984

985

986

987

988

989

990

991

992

993

994

995

996

997

998

999

1000

1001

20. Samanez-Larkin, G. R., Levens, S. M., Perry, L. M., Dougherty, R. F. \& Knutson, B. Frontostriatal white matter integrity mediates adult age differences in probabilistic reward learning. J. Neurosci. Off. J. Soc. Neurosci. 32, 5333-5337 (2012).

21. Samanez-Larkin, G. R., Worthy, D. A., Mata, R., McClure, S. M. \& Knutson, B. Adult age differences in frontostriatal representation of prediction error but not reward outcome. Cogn. Affect. Behav. Neurosci. 14, 672-682 (2014).

22. Hammerer, D. \& Eppinger, B. Dopaminergic and Prefrontal Contributions to Reward-Based Learning and Outcome Monitoring During Child Development and Aging. Dev. Psychol. 48, 826-874 (2012).

23. Bäckman, L., Nyberg, L., Lindenberger, U., Li, S.-C. \& Farde, L. The correlative triad among aging, dopamine, and cognition: Current status and future prospects. Neurosci. Biobehav. Rev. 30, 791-807 (2006).

24. Dreher, J.-C., Meyer-Lindenberg, A., Kohn, P. \& Berman, K. F. Age-related changes in midbrain dopaminergic regulation of the human reward system. Proc. Natl. Acad. Sci. 105, 15106-15111 (2008).

25. Li, S.-C., Lindenberger, U. \& Bäckman, L. Dopaminergic modulation of cognition across the life span. Neurosci. Biobehav. Rev. 34, 625-630 (2010).

26. Schultz, W. Updating dopamine reward signals. Curr. Opin. Neurobiol. 23, 229238 (2013).

27. Schultz, W. Dopamine reward prediction-error signalling: a two-component response. Nat. Rev. Neurosci. 17, 183-195 (2016).

28. Chowdhury, R. et al. Dopamine restores reward prediction errors in old age. Nat. Neurosci. 16, 648-653 (2013). 
1002

1003

1004

1005

1006

1007

1008

1009

1010

1011

1012

1013

1014

1015

1016

1017

1018

1019

1020

1021

1022

1023

1024

1025

29. Engel, C. Dictator games: a meta study. Exp. Econ. 14, 583-610 (2011).

30. Matsumoto, Y., Yamagishi, T., Li, Y. \& Kiyonari, T. Prosocial Behavior Increases with Age across Five Economic Games. PLOS ONE 11, e0158671 (2016).

31. Sze, J. A., Gyurak, A., Goodkind, M. S. \& Levenson, R. W. Greater Emotional Empathy and Prosocial Behavior in Late Life. Emotion 12, 1129-1140 (2012).

32. Ng, T. W. H. \& Feldman, D. C. The relationship of age to ten dimensions of job performance. J. Appl. Psychol. 93, 392-423 (2008).

33. Hubbard, J., Harbaugh, W. T., Srivastava, S., Degras, D. \& Mayr, U. A general benevolence dimension that links neural, psychological, economic, and life-span data on altruistic tendencies. J. Exp. Psychol. Gen. 145, 1351-1358 (2016).

34. Cheung, F. \& Lucas, R. E. When Does Money Matter Most? Examining the Association between Income and Life Satisfaction over the Life Course. Psychol. Aging 30, 120-135 (2015).

35. Buckholtz, J. W. et al. Mesolimbic Dopamine Reward System Hypersensitivity in Individuals with Psychopathic Traits. Nat. Neurosci. 13, 419-421 (2010).

36. White, B. A. Who cares when nobody is watching? Psychopathic traits and empathy in prosocial behaviors. Personal. Individ. Differ. 56, 116-121 (2014).

37. White, S. F. et al. Reduced Amygdala Response in Youths With Disruptive Behavior Disorders and Psychopathic Traits: Decreased Emotional Response Versus Increased Top-Down Attention to Nonemotional Features. Am. J. Psychiatry 169, 750-758 (2012).

38. Marsh, A. A. et al. Empathic responsiveness in amygdala and anterior cingulate cortex in youths with psychopathic traits. J. Child Psychol. Psychiatry 54, 900910 (2013). 
1026

1027

1028

1029

1030

1031

1032

1033

1034

1035

1036

1037

1038

1039

1040

1041

1042

1043

1044

1045

1046

1047

1048

1049

39. Paulhus, D. L., Neumann, C. S., Hare, R. D., Williams, K. M. \& Hemphill, J. F. Self-report Psychopathy Scale 4th Edition (SRP 4) Manual. (MHS, Multi-Health Systems Incorporated, 2017).

40. Leistico, A.-M. R., Salekin, R. T., DeCoster, J. \& Rogers, R. A large-scale metaanalysis relating the Hare measures of psychopathy to antisocial conduct. Law Hum. Behav. 32, 28-45 (2008).

41. Blais, J., Solodukhin, E. \& Forth, A. E. A Meta-Analysis Exploring the Relationship Between Psychopathy and Instrumental Versus Reactive Violence. Crim. Justice Behav. 41, 797-821 (2014).

42. Asscher, J. J. et al. The relationship between juvenile psychopathic traits, delinquency and (violent) recidivism: A meta-analysis. J. Child Psychol. Psychiatry 52, 1134-1143 (2011).

43. Seara-Cardoso, A., Neumann, C., Roiser, J., McCrory, E. \& Viding, E. Investigating associations between empathy, morality and psychopathic personality traits in the general population. Personal. Individ. Differ. 52, 67-71 (2012).

44. Insel, T. R. The NIMH Research Domain Criteria (RDoC) Project: Precision Medicine for Psychiatry. Am. J. Psychiatry 171, 395-397 (2014).

45. Liberman, A. The long view of crime: A synthesis of longitudinal research. (Springer, 2008).

46. Homicide in England and Wales - Office for National Statistics. https://www.ons.gov.uk/peoplepopulationandcommunity/crimeandjustice/articles/ homicideinenglandandwales/yearendingmarch2018\#what-do-we-know-aboutsuspects. 
1050

1051

1052

1053

1054

1055

1056

1057

1058

1059

1060

1061

1062

1063

1064

1065

1066

1067

1068

1069

1070

1071

1072

1073

47. Huchzermeier, C. et al. Are there age-related effects in antisocial personality disorders and psychopathy? J. Forensic Leg. Med. 15, 213-218 (2008).

48. Gill, D. J. \& Crino, R. D. The Relationship between Psychopathy and Age in a Non-Clinical Community Convenience Sample. Psychiatry Psychol. Law 19, 547-557 (2012).

49. Hsieh, S., Schubert, S., Hoon, C., Mioshi, E. \& Hodges, J. R. Validation of the Addenbrooke's Cognitive Examination III in frontotemporal dementia and Alzheimer's disease. Dement. Geriatr. Cogn. Disord. 36, 242-250 (2013).

50. The Psychological Corporation. Wechsler test of adult reading. (Harcourt Assessment, 2001).

51. Huys, Q. J. et al. Disentangling the roles of approach, activation and valence in instrumental and pavlovian responding. PLoS Comput Biol 7, e1002028 (2011).

52. Wittmann, M. K. et al. Global reward state affects learning and activity in raphe nucleus and anterior insula in monkeys. Nat. Commun. 11, 3771 (2020).

53. Zhang, L., Lengersdorff, L., Mikus, N., Gläscher, J. \& Lamm, C. Using reinforcement learning models in social neuroscience: frameworks, pitfalls and suggestions of best practices. Soc. Cogn. Affect. Neurosci. 15, 695-707 (2020).

54. Olsson, A., Knapska, E. \& Lindström, B. The neural and computational systems of social learning. Nat. Rev. Neurosci. 21, 197-212 (2020).

55. Bolenz, F., Kool, W., Reiter, A. M. \& Eppinger, B. Metacontrol of decision-making strategies in human aging. eLife 8, e49154 (2019).

56. Lighthall, N. R., Pearson, J. M., Huettel, S. A. \& Cabeza, R. Feedback-Based Learning in Aging: Contributions and Trajectories of Change in Striatal and Hippocampal Systems. J. Neurosci. 38, 8453-8462 (2018). 
1074

1075

1076

1077

1078

1079

1080

1081

1082

1083

1084

1085

1086

1087

1088

1089

1090

1091

1092

1093

1094

1095

1096

57. Daniel, R., Radulescu, A. \& Niv, Y. Intact Reinforcement Learning But Impaired Attentional Control During Multidimensional Probabilistic Learning in Older Adults. J. Neurosci. 40, 1084-1096 (2020).

58. Nieuwenhuis, S. et al. A computational account of altered error processing in older age: dopamine and the error-related negativity. Cogn. Affect. Behav. Neurosci. 2, 19-36 (2002).

59. Karrer, T. M., Josef, A. K., Mata, R., Morris, E. D. \& Samanez-Larkin, G. R. Reduced dopamine receptors and transporters but not synthesis capacity in normal aging adults: a meta-analysis. Neurobiol. Aging 57, 36-46 (2017).

60. Seaman, K. L. et al. Differential regional decline in dopamine receptor availability across adulthood: Linear and nonlinear effects of age. Hum. Brain Mapp. 40, 3125-3138 (2019).

61. Mayr, U. \& Freund, A. M. Do We Become More Prosocial as We Age, and if So, Why?: Curr. Dir. Psychol. Sci. (2020) doi:10.1177/0963721420910811.

62. Seaman, K. L. et al. Adult age differences in decision making across domains: Increased discounting of social and health-related rewards. Psychol. Aging 31, 737-746 (2016).

63. Rademacher, L., Salama, A., Gründer, G. \& Spreckelmeyer, K. N. Differential patterns of nucleus accumbens activation during anticipation of monetary and social reward in young and older adults. Soc. Cogn. Affect. Neurosci. 9, 825-831 (2014).

64. Gorlick, M. A. et al. Attenuating age-related learning deficits: Emotional valenced feedback interacts with task complexity. Emotion 13, 250-261 (2013). 
1097

1098

1099

1100

1101

1102

1103

1104

1105

1106

1107

1108

1109

1110

1111

1112

1113

1114

1115

1116

1117

1118

1119

65. Lockwood, P. L., O’Nell, K. C. \& Apps, M. A. J. Anterior cingulate cortex: A brain system necessary for learning to reward others? PLOS Biol. 18, e3000735 (2020).

66. Basile, B. M., Schafroth, J. L., Karaskiewicz, C. L., Chang, S. W. C. \& Murray, E. A. The anterior cingulate cortex is necessary for forming prosocial preferences from vicarious reinforcement in monkeys. PLOS Biol. 18, e3000677 (2020).

67. Izuma, K. The social neuroscience of reputation. Neurosci. Res. 72, 283-288 (2012).

68. Andreoni, J. Giving with impure altruism: Applications to charity and ricardian equivalence. J. Polit. Econ. 97, 1447-1447 (1989).

69. Andreoni, J. Impure altruism and donations to public goods: A theory of warmglow giving. Econ. J. 100, 464-477 (1990).

70. Kuss, K. et al. A reward prediction error for charitable donations reveals outcome orientation of donators. Soc. Cogn. Affect. Neurosci. 8, 216-223 (2013).

71. Mobbs, D. et al. A key role for similarity in vicarious reward. Science 324, 900900 (2009).

72. Camerer, C. F. \& Fehr, E. Measuring social norms and preferences using experimental games: A guide for social scientists. Found. Hum. Sociality Econ. Exp. Ethnogr. Evid. Fifteen Small-Scale Soc. 97, 55-95 (2004).

73. Contreras-Huerta, L. S., Lockwood, P. L., Bird, G., Apps, M. A. J. \& Crockett, M. J. Prosocial behavior is associated with transdiagnostic markers of affective sensitivity in multiple domains. Emot. Wash. DC (2020) doi:10.1037/emo0000813. 
1120

1121

1122

1123

1124

1125

1126

1127

1128

1129

1130

1131

1132

1133

1134

1135

1136

1137

1138

1139

1140

1141

1142

74. Lockwood, P. L., Ang, Y.-S., Husain, M. \& Crockett, M. J. Individual differences in empathy are associated with apathy-motivation. Sci. Rep. 7, 17293 (2017).

75. Venegas, J. \& Clark, E. Wechsler Test of Adult Reading. in Encyclopedia of Clinical Neuropsychology (eds. Kreutzer, J. S., DeLuca, J. \& Caplan, B.) 26932694 (Springer, 2011). doi:10.1007/978-0-387-79948-3_1500.

76. Dykiert, D. \& Deary, I. J. Retrospective validation of WTAR and NART scores as estimators of prior cognitive ability using the Lothian Birth Cohort 1936. Psychol. Assess. 25, 1361-1366 (2013).

77. Gordts, S., Uzieblo, K., Neumann, C., Van den Bussche, E. \& Rossi, G. Validity of the Self-Report Psychopathy Scales (SRP-III Full and Short Versions) in a Community Sample. Assessment 24, 308-325 (2017).

78. Neumann, C. S. \& Pardini, D. Factor Structure and Construct Validity of the SelfReport Psychopathy (SRP) Scale and the Youth Psychopathic Traits Inventory (YPI) in Young Men. J. Personal. Disord. 28, 419-433 (2012).

79. Crockett, M. J. et al. Dissociable Effects of Serotonin and Dopamine on the Valuation of Harm in Moral Decision Making. Curr. Biol. 25, 1852-1859 (2015).

80. Lockwood, P. L. et al. Prosocial apathy for helping others when effort is required. Nat. Hum. Behav. 1, 0131 (2017).

81. Lockwood, P. L. \& Klein-Flügge, M. C. Computational modelling of social cognition and behaviour-a reinforcement learning primer. Soc. Cogn. Affect. Neurosci. (2020) doi:10.1093/scan/nsaa040.

82. MacKay, D. J. C. Information Theory, Inference and Learning Algorithms. (Cambridge University Press, 2003). 
1143

1144

1145

1146

1147

1148

1149

1150

1151

1152

1153

1154

1155

1156

1157

1158

1159

1160

1161

1162

1163

1164

1165

83. Stephan, K. E., Penny, W. D., Daunizeau, J., Moran, R. J. \& Friston, K. J. Bayesian Model Selection for Group Studies. Neurolmage 46, 1004-1017 (2009).

84. Daw, N. D., Gershman, S. J., Seymour, B., Dayan, P. \& Dolan, R. J. ModelBased Influences on Humans' Choices and Striatal Prediction Errors. Neuron 69, $1204-1215$ (2011).

85. Palminteri, S., Khamassi, M., Joffily, M. \& Coricelli, G. Contextual modulation of value signals in reward and punishment learning. Nat. Commun. 6, 8096 (2015).

86. Palminteri, S., Wyart, V. \& Koechlin, E. The Importance of Falsification in Computational Cognitive Modeling. Trends Cogn. Sci. 21, 425-433 (2017).

87. R Core Team. R: A language and environment for statistical computing. $R$ Foundation for Statistical Computing. (2017).

88. RStudio Team. RStudio: Integrated Development for R. RStudio, Inc. (2015).

89. Koller, M. robustlmm: An R Package for Robust Estimation of Linear MixedEffects Models. J. Stat. Softw. 75, 1-24 (2016).

90. Bates, D., Mächler, M., Bolker, B. \& Walker, S. Fitting Linear Mixed-Effects Models Using Ime4. J. Stat. Softw. 67, 1-48 (2015).

91. Kassambara, A. rstatix: Pipe-Friendly Framework for Basic Statistical Tests. $R$ package version 0.5.0. (2020).

92. JASP Team. JASP (Version 0.12.2). (2020).

93. Marsman, M. \& Wagenmakers, E.-J. Bayesian benefits with JASP. Eur. J. Dev. Psychol. 14, 545-555 (2017).

94. Tingley, D., Yamamoto, T., Hirose, K., Keele, L. \& Imai, K. mediation: R Package for Causal Mediation Analysis. J. Stat. Softw. 59, 1-38 (2014). 
1167 95. Venables, W. N. \& Ripley, B. D. Modern Applied Statistics with S. (Springer, 1168 2002).

1169 96. Imai, K., Keele, L. \& Tingley, D. A general approach to causal mediation analysis. Psychol. Methods 15, 309-334 (2010).

1171 97. Preacher, K. J., Rucker, D. D. \& Hayes, A. F. Addressing Moderated Mediation 1172 Hypotheses: Theory, Methods, and Prescriptions. Multivar. Behav. Res. 42, 185227 (2007).

1174 98. Judd, C. M. \& Kenny, D. A. Process Analysis: Estimating Mediation in Treatment Evaluations. Eval. Rev. 5, 602-619 (1981). 